\title{
Iniciação Científica na Graduação: O que Diz o Estudante de Medicina?*
}

\author{
Scientific Activities During Undergraduate \\ Education: What do Medical Studente Have to \\ Say?
}

Neilton Araújo de Oliveira ${ }^{l}$

Luiz Anastácio Alves ${ }^{I I}$

Maurício Roberto Luz III

\section{PALAVRAS-CHAVE}

- Ensino Médico;

- Estudantes de Medicina;

- Educação de Graduação em Medicina;

- Pesquisa;

- Medicina.

\section{KEY WORDS:}

- Medical Education:

- Medical Students;

- Undergraduate;

- Research;

- Medicine.
REVISTA BRASILEIRA DE EDUCAÇ̃̃O MÉDICA

\begin{abstract}
RESUMO
A educação médica interessa e repercute em todo o mundo e de forma cada vez mais acentuada, em função da globalização. No Brasil, essa discussão, intensificada com o processo de construção do SUS, foi ampliada a partir das Diretrizes Curriculares Nacionais da Medicina, mas pouco se discute sobre ensino médico e pesquisas. No Enade 2007, 39\% dos alunos informaram iniciação científica regulamentar em seu curso; $12 \%$ declararam existir sem regulamentação e 6,5\% informaram a existência de iniciação científica sem integralização curricular; 10,9\% disseram que não é oferecida e 31\% não souberam opinar. No último Provão, em 2003, 20,9\% dos alunos responderam que a participação em iniciação científica contribuiu para sua formação e 30,7\% declararam não ter participado desse tipo de programa. Este estudo verificou junto aos estudantes do sexto ano, em seis escolas médicas de quatro estados brasileiros, a existência de iniciação científica em seus cursos, a participação ou não nessas atividades e os possíveis motivos para a não participação ou sua inexistência. Mesmo sendo crescente a participação de alunos de graduação em iniciação científica, ainda são muitas as razões para a não realização ou não participação dos alunos. Oitenta e quatro por cento dos alunos defendem a obrigatoriedade da iniciação científica na graduação médica.
\end{abstract}

\begin{abstract}
Medical education is an important issue throughout the world, raising even more interest in the era of globalization. In Brazil, the discussions around medical education increased dramatically during the construction of the Unified Health System and got even more intensive after the establishment of the National Curricular Guidelines. However, very little is known about possible relations and mutual influences between medical education and scientific research. In the 2007 edition of the National Examination of Student Performance - ENADE 2007, 30.7\% of the students from medical schools had not participated in Scientific Initiation activities (a program of research activities for undergraduate students) during their courses, $31 \%$ had no opinion, 39\% of them had participated in such activities and $12 \%$ had done so informally. For $10.9 \%$ of the students there were no Scientific Initiation activities available and $6.5 \%$ reported the existence of such activities however on an extra-curricular basis. It is noteworthy that in 2003 only 20.9\% of the students reported that joining these research activities was important for their medical education while $30.7 \%$ of them reported not participating in research activities In the present study we investigated the availability of Scientific Initiation activities in the courses of sixth year medical students from six medical schools in four Brazilian States. Special attention was paid to the reasons for enrolling or not in the program. Even with an increased participation of students in the program we identified a great number of reasons why students did not participate although $84 \%$ of them consider that participation in the program should be mandatory for medical students in general.
\end{abstract}

“ Trabalho vencedor do PrêmioABEM/2007 de Educação Médica, no 45ํㅡㄹ Congresso Brasileiro de Educação Médica, realizado no período de 20 a 23 de outubro de 2007, em Uberlândia, Minas Gerais, Brasil. (3o Lugar).

${ }^{I}$ Universidade Federal do Tocantins, Tocantins, Brasil; Fundação Oswaldo Cruz, Rio de Janeiro, Brasil.

${ }^{\text {II }}$ Fundação Oswaldo Cruz, Rio de Janeiro, Brasil.

III Universidade Federal do Rio de Janeiro, Rio de Janeiro, Brasil. 


\section{INTRODUÇÃO}

A educação médica passa por modificações de toda ordem, na doutrina e na prática da formação profissional, conectada à contemporaneidade de um mundo globalizado ${ }^{1}$ e condicionada por parâmetros biologicistas, humanísticos e éticos. E, ultimamente, também pelos aspectos políticos e comunitários relacionados às mudanças nos sistemas e serviços de saúde, na implementação do Sistema Único de Saúde (SUS)². O interesse pela transformação e mudanças na educação médica vem aumentando nas duas últimas décadas ${ }^{3,4}$, com o envolvimento de educadores, pesquisadores, gestores, estudantes, profissionais e entidades da área - como o Conselho Federal de Medicina (CFM) e a Associação Brasileira de Educação Médica (Abem) -, além dos ministérios da Saúde (MS) e da Educação (MEC), à medida que cresceu a percepção de que sem profissionais formados com um novo perfil se tornava muito difícil a tarefa de reorganizar modelos de atenção à saúde, conforme preconizado pelo SUS, identificados com os princípios de integralidade, humanização, qualidade da atenção, etc., ${ }^{2,5}$.

Também no âmbito internacional este tema tem merecido maior atenção ${ }^{6-9}$ : eventos como a Segunda Conferência de Edimburgo, o Encontro Internacional de Educação Médica ${ }^{10} \mathrm{e}$ o programa da OMS voltado para a transformação da educação médica ${ }^{11}$ são exemplos desse interesse.

No questionário socioeconômico do Enade 2007, quando perguntados sobre iniciação científica, 39,03\% dos estudantes informaram que há aproveitamento do conhecimento regulamentar para a integralização curricular; $12,2 \%$ informaram que há aproveitamento para a integralização curricular, mas sem regulamentação; e 6,5\% disseram que há, porém sem aproveitamento para a integralização curricular; 10,9\% disseram "não oferece"; e 31,1\% não souberam opinar"12.

Já entre os 390.755 alunos que responderam ao questionário socioeconômico do último Provão, em 2003, 20,9\% acreditam que a participação em programas de iniciação científica contribuiu amplamente para a sua formação e 30,69\% declararam não ter participado desse tipo de programa ${ }^{13}$. Nas duas últimas décadas, diversos autores ${ }^{14-17}$, estudando a relação entre programas de pesquisas na educação médica, defendem claramente e mostram impactos positivos na motivação de estudantes de Medicina, que, por sua vez, têm interesse por pesquisa científica, mas apontam problemas para a não realização de atividades científicas ${ }^{18}$.

Sabe-se que as necessidades de saúde da população, os anseios da sociedade e as demandas do mercado influenciam fortemente o processo de mudança na formação médica. Sendo do Estado a competência de definir as metas da política pública de saúde e, por conseguinte, determinar os objetivos das faculda- des de Medicina como formadoras de profissionais ${ }^{19}$, torna-se relevante investigar o valor de atividades de pesquisa (verificando o interesse e a expectativa que os estudantes têm de delas participarem), principalmente para, em face de sua condição importante na formação médica, induzir a regulamentação urgente da pesquisa científica na graduação em Medicina.

\section{OBJETIVO}

Este estudo teve por objetivo verificar, junto aos estudantes do sexto ano em seis cursos médicos de quatro estados brasileiros, a existência de iniciação científica nesses cursos, a participação ou não nessas atividades de iniciação científica e os possíveis motivos para a não participação ou sua inexistência.

\section{MÉTODOS}

A pesquisa foi realizada entre 2005 e 2007. Após levantamento bibliográfico e documental, aplicamos um questionário para alunos do sexto ano de Medicina $(\mathrm{N}=413)$, em seis escolas médicas (duas particulares e quatro públicas) de quatro estados da federação (uma em Goiás, uma em Alagoas, uma no Rio Grande do Sul e três no Rio de Janeiro). A distribuição dos alunos e o número de alunos que respondeu ao questionário estão mostrados na Tabela 1.

TABELA 1

Cursos médicos/alunos do sexto ano médico - questionários respondidos $\left(n^{\circ} e \%\right)$

\begin{tabular}{lcccc}
\hline $\begin{array}{c}\text { Curso médico } \\
\text { (público ou } \\
\text { privado) }\end{array}$ & Localização & Concluintes & $\begin{array}{c}\text { Questionários } \\
\text { respondidos }\end{array}$ & $\%$ \\
\hline $1-$ público & capital & 107 & 91 & 85,04 \\
$2-$ público & capital & 80 & 67 & 83,75 \\
3 - público & capital & 153 & 98 & 64,05 \\
$4-$ público & capital & 88 & 61 & 69,31 \\
$5-$ privado & interior & 73 & 24 & 32,87 \\
6- privado & capital & 100 & 72 & 72,00 \\
\hline Total: 6 cursos & & 601 & 413 & 68,71 \\
\hline
\end{tabular}

Fonte: MEC/Inep - Censo 2005.

Entre os pontos investigados, verificamos a existência de atividades de iniciação científica em seus cursos médicos, a participação ou não dos alunos nessas atividades e as possíveis razões para a sua não participação ou sua inexistência. Também foram realizadas entrevistas semi-estruturadas com alguns alunos dessas escolas, buscando qualificar as respostas quantitativas obtidas pelo questionário. Os dados foram tabulados e comparados com os obtidos no Provão 2003 e no 
TABELA 2

Perfil socioeconômico dos alunos (sexto ano médico) pesquisados (\%)

Brasil 2005 - 2007

\begin{tabular}{|c|c|c|c|c|c|c|c|c|}
\hline Item & Parâmetro & $\begin{array}{c}\text { Total } \\
\mathrm{N}=413 \\
\%\end{array}$ & $\begin{array}{l}\text { Curso } 1 \text { n } \\
=91\end{array}$ & $\begin{array}{c}\text { Curso } 2 \mathrm{n} \\
=67\end{array}$ & $\begin{array}{c}\text { Curso } 3 \mathrm{n} \\
=98\end{array}$ & $\begin{array}{c}\text { Curso } 4 \mathrm{n} \\
=61\end{array}$ & $\begin{array}{l}\text { Curso } 5 \\
N=24\end{array}$ & $\begin{array}{c}\text { Curso } 6 \\
N=72\end{array}$ \\
\hline \multirow{3}{*}{ Idade } & 20 a 22 & 12 & 9 & 22 & 13 & 11 & 9 & 8 \\
\hline & 23 a 25 & 63 & 59 & 66 & 59 & 64 & 65 & 67 \\
\hline & + de 25 & 25 & 32 & 12 & 28 & 26 & 26 & 25 \\
\hline \multirow{2}{*}{ Sexo } & masc. & 42 & 54 & 30 & 35 & 42 & 52 & 42 \\
\hline & fem. & 58 & 46 & 70 & 65 & 58 & 48 & 58 \\
\hline \multirow{3}{*}{ Estado civil } & solteiro & 88 & 85 & 96 & 94 & 82 & 91 & 92 \\
\hline & casado & 12 & 15 & 4 & 6 & 26 & 9 & 8 \\
\hline & outro & 3 & 0 & 0 & 0 & 18 & 0 & 0 \\
\hline \multirow{5}{*}{ Cor } & negra & 4 & 4 & 4 & 1 & 12 & 0 & 0 \\
\hline & parda & 24 & 47 & 49 & 9 & 7 & 27 & 7 \\
\hline & amarela & 3 & 3 & 6 & 1 & 4 & 0 & 3 \\
\hline & branca & 67 & 44 & 39 & 87 & 77 & 68 & 90 \\
\hline & outra & 1 & 1 & 1 & 1 & 0 & 5 & 0 \\
\hline \multirow{2}{*}{ Mora com a família } & $\operatorname{sim}$ & 70 & 69 & 85 & 69 & 84 & 25 & 58 \\
\hline & não & 30 & 31 & 15 & 31 & 16 & 75 & 42 \\
\hline \multirow{2}{*}{ Em imóvel } & próprio & 69 & 66 & 76 & 67 & 78 & 17 & 75 \\
\hline & alugado & 31 & 34 & 24 & 33 & 22 & 83 & 25 \\
\hline \multirow{2}{*}{$\begin{array}{l}\text { Tem outro curso } \\
\text { superior }\end{array}$} & $\operatorname{sim}$ & 17 & 0 & 1 & 25 & 64 & 0 & 3 \\
\hline & não & 83 & 100 & 99 & 75 & 36 & 100 & 97 \\
\hline \multirow{2}{*}{$\begin{array}{l}\text { Trabalho/ outra } \\
\text { atividade }\end{array}$} & $\operatorname{sim}$ & 25 & 5 & 9 & 36 & 66 & 13 & 13 \\
\hline & não & 75 & 95 & 91 & 64 & 34 & 88 & 88 \\
\hline \multirow{5}{*}{ Renda familiar } & até $5 \mathrm{sm}$ & 2 & 0 & 7 & 6 & 0 & 0 & 0 \\
\hline & 5 a $10 \mathrm{sm}$ & 8 & 7 & 16 & 8 & 5 & 18 & 3 \\
\hline & 10 a $15 \mathrm{sm}$ & 16 & 11 & 19 & 25 & 16 & 32 & 4 \\
\hline & 15 a $20 \mathrm{sm}$ & 26 & 29 & 22 & 26 & 34 & 23 & 17 \\
\hline & + de $20 \mathrm{sm}$ & 48 & 54 & 34 & 35 & 45 & 27 & 76 \\
\hline \multirow{4}{*}{ Escolaridade do pai } & $1^{\mathrm{o}} \mathrm{g}$ & 6 & 5 & 6 & 7 & 4 & 13 & 3 \\
\hline & $2^{\circ} \mathrm{g}$ & 19 & 25 & 34 & 18 & 12 & 8 & 10 \\
\hline & $3^{\circ} \mathrm{g}$ & 50 & 45 & 40 & 58 & 65 & 46 & 44 \\
\hline & pós-gr. & 25 & 24 & 19 & 18 & 19 & 33 & 43 \\
\hline \multirow{4}{*}{ Escolaridade da mãe } & $1^{\mathrm{o}} \mathrm{g}$ & 4 & 0 & 7 & 9 & 4 & 4 & 0 \\
\hline & $2^{\circ} \mathrm{g}$ & 24 & 31 & 25 & 25 & 22 & 25 & 18 \\
\hline & $3^{\circ} \mathrm{g}$ & 49 & 46 & 48 & 44 & 53 & 38 & 60 \\
\hline & pós-gr. & 23 & 23 & 19 & 22 & 22 & 33 & 22 \\
\hline \multirow{2}{*}{ Possui computador } & $\operatorname{sim}$ & 94 & 90 & 100 & 96 & 96 & 75 & 94 \\
\hline & não & 6 & 10 & 0 & 4 & 4 & 25 & 6 \\
\hline \multirow{2}{*}{ Acessa internet } & $\operatorname{sim}$ & 98 & 97 & 96 & 99 & 100 & 96 & 100 \\
\hline & não & 2 & 3 & 4 & 1 & 0 & 4 & 0 \\
\hline \multirow{2}{*}{ Possui carro } & $\operatorname{sim}$ & 63 & 70 & 63 & 52 & 77 & 25 & 67 \\
\hline & não & 37 & 30 & 37 & 48 & 23 & 75 & 33 \\
\hline \multirow{2}{*}{ Família tem carro } & $\operatorname{sim}$ & 97 & 96 & 97 & 93 & 100 & 92 & 100 \\
\hline & não & 3 & 4 & 3 & 7 & 0 & 8 & 0 \\
\hline \multirow{5}{*}{$\begin{array}{l}\text { Quantidade de livros } \\
\text { que possui }\end{array}$} & até 50 & 31 & 48 & 34 & 24 & 5 & 54 & 36 \\
\hline & 50 a 100 & 27 & 34 & 30 & 20 & 32 & 17 & 22 \\
\hline & 100 a 200 & 23 & 12 & 18 & 31 & 43 & 17 & 17 \\
\hline & 200 a 500 & 10 & 3 & 6 & 16 & 12 & 13 & 14 \\
\hline & + de 500 & 8 & 2 & 12 & 9 & 7 & 0 & 11 \\
\hline
\end{tabular}

Total de alunos $(\mathrm{N})=413$. "sm" = salário mínimo

Tabela preparada a partir de dados/respostas aos questionários aplicados. 
Enade 2007. Todos os sujeitos que participaram do estudo assinaram um termo de consentimento livre e esclarecido, e o projeto de pesquisa obteve aprovação do Comitê de Ética e Pesquisa do IOC-Fiocruz (Parecer 302-06).

\section{RESULTADOS E DISCUSSÃO}

Neste estudo, que tem caráter exploratório, entendemos que múltiplos fatores podem contribuir para o desempenho dos estudantes de Medicina na graduação. Além de critérios socioeducativos, culturais e econômicos dos alunos (Tabela 2), outros devem ser considerados, como a qualificação docente, logística e organização do curso, estrutura curricular e integração ensino-serviços de saúde, por exemplo. No entanto, presumimos e destacamos, especialmente, a participação desses alunos em atividades de pesquisa, nas instituições em que estudam, como fator relevante no processo de ensino-aprendizagem na Medicina (ver Tabela 2).

Das repostas dos alunos ao questionário aplicado nos seis cursos médicos, destacamos diferentes questões relacionadas às atividades de pesquisa, especialmente a participação dos alunos na iniciação científica. Nesse particular, também verificamos e analisamos as possíveis razões para a não existência e/ou não participação dos estudantes nessas atividades durante a sua formação. O questionário completo deste trabalho e respectivas respostas (em números absolutos e percentuais) estão disponibilizadas no site http://www.educacaomedica. org.br para consulta dos interessados.

Quando perguntamos aos 413 alunos se haviam participado ou participavam de programas de iniciação científica, 28\% não participaram; $37 \%$ participaram em disciplina optativa; $19 \%$ em disciplina obrigatória; $12 \%$ em atividades fora de sua escola e $4 \%$ deram outras respostas. No Provão 2003, 30\% dos alunos que responderam ao questionário declararam não ter participação em pesquisas; no Enade 2007,39\% dos alunos informaram iniciação científica regulamentar em seu curso.

De maneira geral, é crescente o interesse dos alunos de Medicina em participar de iniciação científica durante a graduação - apenas 7\% deles não têm interesse por pesquisas. Das seis escolas pesquisadas, duas apresentam programas de iniciação científica estruturados e é aí que se concentram os maiores percentuais de alunos que participam de atividades de iniciação científica. São diversas as razões pelas quais os alunos dizem não participar dessas atividades, indo desde a falta de interesse do aluno, em percentuais menores, até a inexistência de pessoal qualificado ou motivado, em percentuais médios, ou, em percentuais mais elevados, à carência de condições materiais ("d") e, principalmente, à falta de estímulo institucional ("c") para a realização de pesquisas.
Esta última parece ser a dificuldade maior para a participação desses alunos nessas atividades, mesmo onde o programa de iniciação científica existe oficialmente. Em dois dos seis cursos investigados, 33,3\% e 34,5\% dos alunos informaram que a principal razão para não participarem de pesquisas é a carência de estrutura física e material, enquanto nos quatro outros cursos 39,3\%, 45,9\%, 49,5\% e 50,0\% dos alunos declararam como principal razão a falta de estímulo por parte da instituição, sendo esta também a principal causa quando se analisa o conjunto dos alunos $(38,4 \%)$.

TABELA 3

Razão mais importante para a não realização de atividades de pesquisa científica

\begin{tabular}{llll}
\hline Todos & $38,4 \%$ & c & $1^{\text {a }}$ opção (mais importante) \\
Curso 1 & $49,5 \%$ & c & $1^{\text {a }}$ opção (mais importante) \\
Curso 2 & $39,3 \%$ & c & $1^{\text {a }}$ opção (mais importante) \\
Curso 3 & $34,5 \%$ & d & $1^{\text {a }}$ opção (mais importante) \\
Curso 4 & $45,9 \%$ & c & $1^{\text {a }}$ opção (mais importante) \\
Curso 5 & $50,0 \%$ & c & $1^{\text {a }}$ opção (mais importante) \\
Curso 6 & $33,3 \%$ & d & $1^{\text {a }}$ opção (mais importante) \\
\hline
\end{tabular}

Do total de alunos, $84 \%$ defendem a obrigatoriedade da iniciação científica na graduação médica. Ao se comparar escola privada e escola pública do mesmo estado ou escolas públicas dos demais estados, a variação na participação de alunos nessas escolas parece se dar em função da existência de programas de iniciação científica e/ou da presença maior de grupos de pesquisas em suas instituições. As escolas públicas apresentam índices maiores de alunos que participam de atividades de pesquisa, o que era esperado, já que a maior parte dos grupos de pesquisa está em escolas públicas ${ }^{20}$. Possivelmente, encontraremos resultados diferentes numa amostra com maior número de escolas públicas e privadas.

Montes $^{21}$, comentando sobre a inserção da disciplina de iniciação científica no currículo da USP, implantado em 1998, ressalta que “...os esforços (bem sucedidos) para melhorar os programas de pós-graduação e o nível de pesquisa deixaram a impressão de que a graduação havia sido relegada a um segundo plano...", mas conclui que o novo currículo, contendo atividades de pesquisa científica, estimula o aluno à compreensão, logo no início da carreira, da correlação entre ensino, pesquisa e extensão. Nos períodos profissionalizantes a correlação é com a extensão e os serviços de saúde, porém “...nas áreas básicas resulta claro que essa correlação é com a pesquisa".

Dentre as diversas e diferentes falas dos alunos, durante as entrevistas, separamos algumas que exemplificam o atual 
contexto de participação em programas de iniciação científica nos cursos de graduação em medicina:

Só entendi melhor essa questão de pesquisa depois que passei um tempo num projeto e vi junto com outros colegas as muitas vantagens da investigação científica... mesmo eu ainda tendo dificuldade de lidar com alguns aspectos científicos, vou continuar me aproximando de grupos de pesquisa... mas não quero ser cientista!

Segundo Dini e Batista ${ }^{22}$, a expectativa central de todos os alunos, durante todo o curso de medicina, é ser um bom médico, mas uma visão idealista inicial vai progressivamente mudando para uma visão mais realista da profissão e no início do $6^{\circ}$ ano sua expectativa está totalmente voltada para o sucesso nas seleções de residências. O que poderíamos discutir é se nessa visão de bom médico estaria incluído, intrinsecamente, atividades de pesquisas e, ainda, perguntar: como gostar e entender o papel importante da pesquisa na preparação de um "bom médico", se o aluno não tiver oportunidade de iniciação científica e durante sua evolução no curso não continuar tendo contado com a pesquisa científica.

Eu até que gosto de pesquisa, só que no meu curso não tem, aí só posso participar se for fora da minha escola.

O binômio ensino-pesquisa é considerado, de forma unânime, uma conquista permanente e intrínseca do conceito de universidade e, mesmo os que o criticam o fazem por, sendo considerado necessário, se mostra insuficiente ${ }^{23}$. Então, apesar dessa oferta de atividades de pesquisa nos cursos médicos ainda constituir situações pontuais e minoritárias, e em alguns casos quase que extraordinárias, está aumentando a compreensão de que deve ser uma atividade articulada ao ensino e integrada ao currículo regular.

Nas duas vezes que participei de atividade científica em um projeto de pesquisa, eu acho que aprendi mais fácil... mas isso não é muito freqüente no meu curso, então desanima!...

Um pressuposto e requisito essencial à boa prática médica é a habilidade para pensar cientificamente e usar o método científico, o que significa, em conseqüência, que o médico está submetido a uma necessidade de adquirir continuamente novas informações científicas e novos conhecimentos ${ }^{24}$.
Portanto, a tendência de envolver alunos de graduação da escola médica cada vez mais em programas de pesquisa e iniciação científica, corresponde a uma expectativa por parte dos estudantes ${ }^{25}$ e fica evidenciada, nesse trabalho, na medida que apenas $7 \%$ dos estudantes não têm interesse por pesquisas.

\section{CONCLUSÃO}

A dinâmica do processo de mudanças na formação médica, atualmente, além de muito intensa, é determinada por múltiplos fatores que precisam ser discutidos e articulados, em seu conjunto. Portanto, nenhuma medida isoladamente pode ser considerada como a responsável pelo sucesso de uma proposta inovadora, entretanto, apesar do caráter exploratório deste trabalho, ousamos dizer que a participação do aluno em Programas de Iniciação Científica é estratégica e deve ser estimulada e priorizada.

Cerca de três quartos dos estudantes manifestaram que a pesquisa científica é uma experiência institucional importante e quase todos concordaram com a possibilidade de ser obrigatória a atividade de iniciação científica na formação médica. Tal situação, que está em consonância com estudos internacionais, demonstra a atualidade da proposta de regulamentação da pesquisa como parte do currículo médico no Brasil.

Mesmo sendo um princípio das Diretrizes Curriculares Nacionais ${ }^{26}$, a realização de pesquisa na formação médica ainda é pouco freqüente e precisa ser conhecida com mais profundidade para que se possa compreender sua influência, principalmente para tentar estabelecer uma medida que indique claramente o valor da correlação entre participação em iniciação científica e aprendizagem.

Inexistência de pessoal capacitado e com tempo disponível para orientação de trabalhos, carência de estrutura física e falta de estímulo institucional são os maiores desafios.

\section{REFERÊNCIAS}

1. Arruda BKG org. A educação profissional em saúde e a realidade social. Recife: Instituto Materno Infantil de Pernambuco (IMIP)/ Ministério da Saúde; 2001.

2. Brasil. Constituição, 1988. Constituição da República Federativa do Brasil. Brasília: Senado Federal; 1988.

3. Brasil. Ministério da Saúde. Conselho Nacional de Saúde. O Desenvolvimento do Sistema Único de Saúde: avanços, desafios e reafirmação dos seus princípios e diretrizes / MS, Conselho Nacional de Saúde. Brasília: Ministério da Saúde; 2002.

4. Stella RCR. Conquistas e Desafios [editorial]. Rev Bras Educ Med 2001; 25(3):5-6 
5. Paim JS. Recursos Humanos em Saúde no Brasil: problemas crônicos e desafios agudos. São Paulo: AdSaúde; 1994. (Série Temática 1. Fac. de Saúde Pública/USP).

6. Brotherton SE, Rochey PH, Etzel SI. US Graduate Medical Education 2003-2004. JAMA 2004; 292:1032-37.

7. Lempp H, Seale $C$. The hidden curriculum in undergraduate medical education: qualitative study of medical students' perceptions of teaching. BMJ 2004; 329:770-73.

8. Smits PBA, Verbeek JHAM, Nauta MCE, Tem Cate ThJ, Metz JCM, Van Dijk FJH. Factors predictive of successful learning in postgraduate medical education. Med. Educ. 2004; 38:848-51.

9. Ferguson E, James D, Madeley L. Factors associated with success in medical school: systematic review of the literature. BMJ 2002 324: 952-57.

10. Organização Pan-Americana de Saúde. Anais do Encontro Continental de Educação Médica. Montivideo: OPS/OMS; 1997.

11. World Health Organization. Changing medical education and pratice: an agenda for action. Genebra; 1991.

12. Instituto Nacional de Estudos e Pesquisas Educacionais Anísio Teixeira, 2007. [capturado 28 jul. 2007]. Disponível em: http://www.inep.gov.br/informativo/2007/ed_168.htm

13. Instituto Nacional de Estudos e Pesquisas Educacionais Anísio Teixeira. Relatório-síntese. Relatório Síntese do ENC-Provão 2003. [capturado 4 set 2004]. Disponível em: http://www.inep.gov.br/superior/provao/sintese/sintese.htm.

14. Yamamoto L. The role of research in medical education. Hawaii Medical Journal 1999; 58:25-6.

15. Solomon SS, Tom SC, Pichert J, Wasserman D, Powers AC. Impact of Medical Student Research in the Development of Physician-Scientists. J Inv Med 2003; 51(3):149-56.

16. Jacobs CD, Cross PC. The value of medical student research: the experience at Stanford University School of Medicine. Med Educ 1995;29:342-6.

17. Houlden RL, Raja JB, Collier CP, Clark AF, Waugh JM. Medical students'perceptions of an undergraduate research elective. Med Teach 2004;26(7): 659-61.

18. Pereyra WJF, Petroianu A. Medical students interest in scientific research. Rev Bras Educ Med [on line]. 2000;24(2) [capturado 7 set. 2006]; 9-13. Disponível em: www.abem- educmed.org.br/paginasrevistas/rbem/24/rbemv24_n2_ artigo.pdf.

19. Bulcão LG, El-Kareh AC, Sayd JD. Ciência e ensino médico no Brasil (1930-1950). Hist. cienc. saude-Manguinhos [on line]. 2007;14(2) [capturado6 set. 2007]; p-p. Disponível em: http:/ / www.scielo.br/scielo.php?script=sci_arttext\&pid=S01049702007000200005\&lng=pt\&nrm=iso\&tlng=pt.

20. Conselho Nacional de Desenvolvimento Científico e Tecnológico. Diretório dos Grupos de Pesquisa no Brasil [on line] 2004; [capturado 7 set. 2006]. Disponível em: http:// lattes.cnpq.br/diretorio/.

21. Montes GS. Da importância de uma disciplina de iniciação científica ao currículo nuclear na graduação em medicina na USP. Rev. Bras. Card. [online] 2000; [capturado 4 out 2006]; 2(2): 70-7. Disponível em: http://med.fm.usp.br/ pcm/artigo.htm.

22. Dini OS, Batista NA. Graduação e Prática Médica: Expectativas e Concepções de Estudantes de Medicina do $1^{\circ}$ ao $6^{\circ}$ ano. Rev. Bras. Educ. Méd. 2004; 28(3)

23. Castro LP. A pesquisa no Hospital Universitário. Conferência no Congresso da SBPC de 1997, Belo HorizonteMG.Universidade Federal de Minas Gerais-Faculdade de Medicina. [capturado 1 jul 2005]. Disponível em: http:// www.medicina.ufmg.br/cememor/depoim5.htm.

24. CCIIME-Core Committee, I. f. I. M. E. Global minimum essential requirements in medical education. Med Teach 2002; 24(2): 130-5.

25. Cardoso GP, Silva Junior CT, Martinho JMSG, Cyrillo RJT. Iniciação científica em medicina: uma questão de interesse para todas as especialidades. Pulmão RJ 2004; 13(1): 8-12.

26. Almeida M org. Diretrizes Curriculares Nacionais para os Cursos Universitários da área da saúde. Londrina: Rede Unida; 2003.

\section{CONFLITOS DE INTERESSE}

Declarou não haver

\section{ENDEREÇO PARA CORRESPONDÊNCIA}

Neilton Araujo de Oliveira

(NEST-UFT): 109 Norte,

Av. NS 15, ALCNO 14, Bloco IV, sala 205.

CEP. 77001-090 - Palmas - TO

E-mail: neilton@uft.edu.br ou nest@uft.edu.br. 